\title{
金属锂电极固液界面中关键的内亥姆霍兹层
}

刘忠范

北京大学化学与分子工程学院, 北京 100871

\section{The Pivotal Inner Helmholtz Plane for Solid Electrolyte Interphase in Lithium Metal Anodes}

\section{LIU Zhongfan}

College of Chemistry and Molecular Engineering, Peking University, Beijing 100871, P. R. China.

Email: zfliu@pku.edu.cn.

Published online: June 13, 2019.

(a)

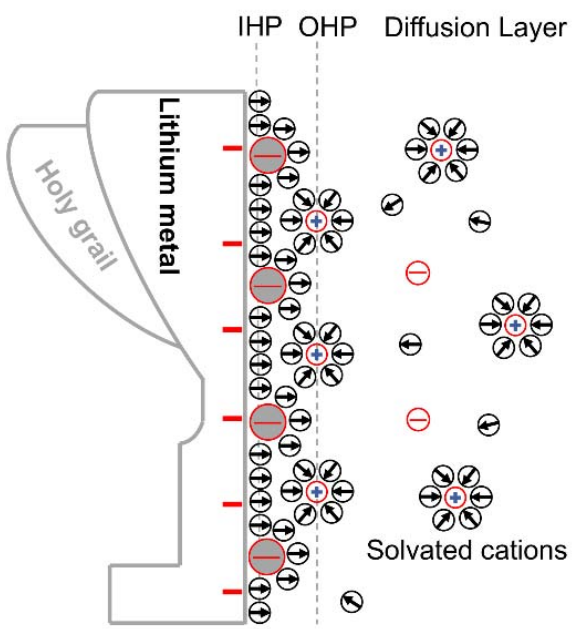

(b)

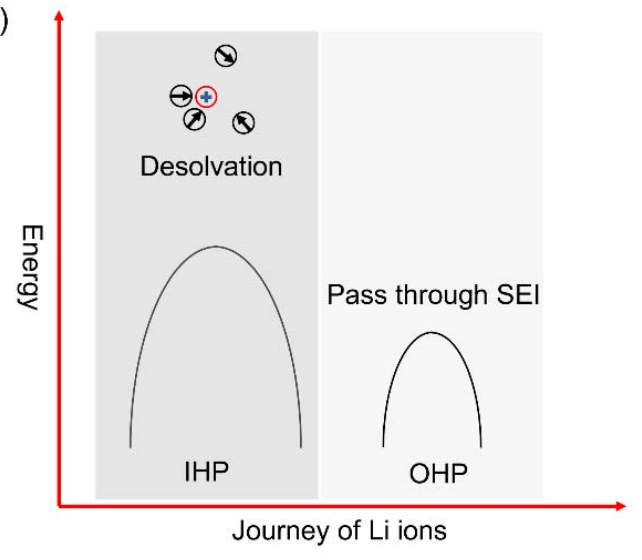

specifically absorbed anions $\Theta$ solvents $\oplus$ Li ions

(a)金属锂表面双电层结构; (b)特性吸附阴离子诱导的固液界面对锂离子输运的影响。

电极中的固体电解质界面膜 (solid electrolyte interphase, SEI)由以色列希伯来大学Peled教授在 1979年提出 ${ }^{1}$, 最初是用来表示金属锂负极在有机 电解液中形成的固液界面膜, 后来延伸到其他负 极材料体系(如石墨负极 2 、硅负极、锌负极3等)。SEI 膜概念的提出和发展在锂金属电池(lithium metal batteries, LMBs)、锂离子电池(lithium ion batteries, LIBs)及其他储能电池体系的研究中都发挥着重 要作用。

SEI膜形成过程涉及到复杂的固液表界面反 应, 既包括自发的化学反应过程又涵盖充放电过
程中的电化学反应过程 ${ }^{4}$ 。因此, 科学地理解固液 界面形成机制和影响因素对设计和调控SEI具有 重要意义。近些年, 研究者发现电解液的溶剂化行 为对于 SEI膜的稳定性具有重要影响 ${ }^{5}$, 尤其是在 高浓度盐条件下的独特溶剂化行为能够诱导生成 稳定的 $\mathrm{SEI}^{6,7}$ 。然而, 电极表面的双电层结构 ${ }^{8}$, 尤 其是双电层中的内亥姆霍兹层的特性离子吸附在 SEI膜的形成中发挥的作用往往被忽视(图a), 相关 的基础研究进展也较少报道。

最近, 针对以上问题, 北理工大学黄佳琦教授 课题组深入探究了以金属锂负极为典型代表的电 
解固液界面形成过程, 相关成果发表于Journal of

the American Chemical Society 上 $^{9}$ 。该研究工作认 为电极表面双电层结构中内亥姆霍兹层的特性阴 离子吸附和外亥姆霍兹层及电解液体相的溶剂化 行为共同决定了SEI的成分和结构, 进而影响电池 的电化学性能。文章通过巧妙的设计保证了电解 液体相具有相同的离子溶剂化结构, 然后把研究 重点聚焦在内亥姆霍兹层的特性吸附上, 通过模 拟计算验证了硝酸根 $\left(\mathrm{NO}_{3}^{-}\right)$阴离子在硝酸根 $\left(\mathrm{NO}_{3}^{-}\right)$、氟离子 $\left(\mathrm{F}^{-}\right)$、双氟亚胺离子 $\left(\mathrm{FSI}^{-}\right)$和乙二醇 二甲醚(DME)等多种组分竞争吸附环境下的优势 吸附, 并在电化学测试中的非法拉第过程和法拉 第过程中发现了相关证据。

该研究团队同时探究了特性阴离子吸附通过 电化学变化形成的SEI对于锂沉积形貌的影响, 发 现 $\mathrm{NO}_{3}^{-}$诱导产生的 SEI能够促进金属锂的球状沉 积, 完全不同于常规认知的枝晶状沉积, 该沉积行 为的差异使得匹配磷酸铁锂正极的金属锂电池寿 命至少延长了 11 倍, 组装的软包电池在 80 次循环 后依旧保持 $99.8 \%$ 的容量。在深入探究锂离子在固 液界面输运的活化能等动力学因素后, 研究人员 认为内亥姆霍兹层的特性吸附主要影响锂离子的 脱溶剂化能(包括穿越SEI过程中的脱溶剂化), 外 亥姆霍兹层的溶剂化行为主要影响锂离子穿过 SEI的能垒(图b)。

该观点的提出具有重要的创新性和前瞻性, 构 建了固液界面形成过程的完整链条, 并推动了固 液界面的动力学相关研究。该系列工作得到了国家
自然科学基金委、科技部国家重点研发计划、北京 市科委项目的经费支持。

\section{References}

(1) Peled, E. J. Electrochem. Soc. 1979, 126, 2047. doi: $10.1149 / 1.2128859$

(2) Noel, M.; Santhanam, R. J. Power Sources 1998, 72, 53. doi: 10.1016/S0378-7753(97)02675-X

(3) Bass, K.; Mitchell, P. J.; Wilcox, G. D.; Smith, J. J. Power Sources 1991, 35, 333. doi: 10.1016/0378-7753(91)80117-g

(4) Yan, C.; Cheng, X. B.; Yao, Y. X.; Shen, X.; Li, B. Q.; Li, W. J.; Zhang, R.; Huang, J. Q.; Li, H.; Zhang, Q. Adv. Mater. 2018, 30, 1804461. doi: 10.1002/adma.201804461

(5) Yan, C.; Yao, Y. X.; Chen, X.; Cheng, X. B.; Zhang, X. Q.; Huang, J. Q.; Zhang, Q. Angew. Chem. Int. Ed. 2018, 57, 14055. doi: 10.1002/anie.201807034

(6) Yamada, Y.; Furukawa, K.; Sodeyama, K.; Kikuchi, K.; Yaegashi, M.; Tateyama, Y.; Yamada, A. J. Am. Chem. Soc. 2014, 136, 5039. doi: $10.1021 / \mathrm{ja} 412807 \mathrm{w}$

(7) Suo, L.; Borodin, O.; Gao, T.; Olguin, M.; Ho, J.; Fan, X.; Luo, C.; Wang, C.; Xu, K. Science 2015, 350, 938. doi: $10.1126 /$ science.aab1595

(8) Borodin, O.; Ren, X.; Vatamanu, J.; von Wald Cresce, A.; Knap, J.; Xu, K. Acc. Chem. Res. 2017, 50, 2886. doi: $10.1021 /$ acs.accounts. $7 b 00486$

(9) Yan, C.; Li, H. R.; Chen, X.; Zhang, X. Q.; Cheng, X. B.; Xu, R.; Huang, J. Q.; Zhang, Q. J. Am. Chem. Soc. 2019, 141, 9422. doi: $10.1021 /$ jacs.9b05029 